\title{
Practical Aspects for Reliable Standardless Quantification in Energy Dispersive X- ray Spectrometry
}

Philippe Pinard ${ }^{1}$, Simon Burgess ${ }^{1}$, Alan Protheroe ${ }^{1}$, Rosie Jones ${ }^{2}$ and Peter Statham ${ }^{1}$

${ }^{1}$ Oxford Instruments NanoAnalysis, High Wycombe, England, United Kingdom, ${ }^{2}$ Oxford Instruments, High Wycombe, England, United Kingdom

As for any electron microprobe quantitative analysis (EPMA), standardless quantification offered by energy dispersive X-ray spectrometry (EDS) systems requires a careful sample preparation and a detailed experimental procedure to obtain accurate results [1]. Previous publications [2-6] have shown that one approach to standardless quantification can achieve unnormalized results with an accuracy better than 5\%, without the need to store, maintain and measure standards. In this work, we place emphasis on the experimental procedure to achieve this level of accuracy in any scanning electron microscope (SEM).

Our approach to standardless quantification, TruQ, relies on (1) a "factory" standards database, where several standards were carefully validated and measured, (2) an accurate model of the efficiency of any detector, (3) correction of the energy scale, escape and sum peaks and pile-up losses, (4) removal of the background using a convolution filter, (5) calculated peak profiles that take into account the acquisition conditions, detector characteristics and X-ray emission physics, and (6) the XPP matrix correction [7]. More details about these components are found in [2-6].

While each microscope and sample are different, we propose a generic experimental protocol to quantify unknown samples consisting of heavy elements $(\mathrm{Z} \geq \mathrm{Na})$ or oxides where oxygen can be quantified by stoichiometry.

1. Ensure that the unknown sample is flat, polished and conductive. A carbon coating of 10-20nm in thickness normally makes most samples conductive.

2. Prepare a pure metal sample (e.g. $\mathrm{Cu}, \mathrm{Co}$ ). Placing a piece of $\mathrm{Cu}$ tape on a stub works well.

3. Insert both samples in the SEM. It is advantageous to adjust the height of the samples, so they are roughly at the same level.

4. Adjust the accelerating voltage to $20 \mathrm{kV}$. This is normally a good starting point to excite a quantifiable X-ray line for all elements.

5. Move the sample to the recommended working distance of the ED detector. Set the working distance on the microscope, remove lens hysteresis and move the z-position of the stage to bring the sample into focus. Any deviation in the sample height effectively modifies the take-off angle of the detector and introduces an error in the absorption correction. Note that this must be repeated for each sample.

6. Make sure the field of view is smaller than $0.5 \mathrm{~mm}$ to avoid any occlusion by the detector collimator. The electron beam should be in spot mode or scanning over a small area of the sample. EPMA cannot determine the average composition of a sample by scanning over its entire surface. The matrix correction requires the sample to be homogeneous within the interaction volume.

7. Ensure the beam current stability is better than $1 \%$. This can be achieved by acquiring a spectrum every 30 s for $5 \mathrm{~min}$ on the pure metal sample and monitoring the intensity of the highest energy Xray line series (e.g. $\mathrm{Cu} \mathrm{K}$ ). For example, collecting a spectrum with 600,000 counts at $20 \mathrm{kV}$ on a 
pure $\mathrm{Cu}$ sample takes less than $5 \mathrm{~s}$ with an input count rate (ICR) of 200kcps and results in a relative error on the beam current measurement of approx. $0.3 \%$.

8. Move to the unknown sample and adjust the acquisition conditions. As shown below, an ICR between 50 and 200kcps gave accurate quantification results with the silicon drift detector (SDD) used. As a rule of thumb, the process time should be adjusted to achieve the best spectral resolution while keeping the dead time below $50 \%$ for a given ICR.

9. Perform a beam measurement on the pure metal sample. As several types of SEMs are not equipped with an ammeter, this is a practical and easy alternative to a Faraday cup. It takes less than 20s to acquire 600,000 counts in the spectrum at the recommended ICRs. It is good practice to perform beam measurements throughout the microscope session.

10. Acquire a spectrum on the unknown sample. Acquiring at least 1 million counts in the spectrum is a good starting point. Depending on the element with the lowest detectable concentration, the number of counts may need to be increased to reduce its statistical uncertainty.

11. Verify the automatic element identification using the fitted spectrum (an overlay of the fitted peak profiles and simulated background), the simulated theoretical spectrum (based on the acquisition conditions and the calculated composition [8]) and the analytical total. EPMA will never yield accurate results if the elements in the sample are not correctly identified.

In order to verify the standardless quantification on a given system, it is recommended to first measure a sample with a known composition using the same collection parameters to be used to analyse your samples (e.g. mineral standard, stainless steel). Any misidentification of elements or deviation in the concentrations may indicate issues with the system, such as sample charging, incorrect geometry, etc.

Using this protocol, we evaluated the accuracy of our approach to standardless quantification using 49 standards, consisting of pure elements, simple binaries and minerals, using an Ultim Max 100 SDD installed on a Leo 1450VP at two accelerating voltages (15 and 20kV) and two ICRs (50 and 200kcps). The ICR was adjusted on Co, which was also used for the beam measurements. The acquisition time (i.e. real time) was less than $30 \mathrm{~s}$ at $50 \mathrm{kcps}$ and less than $8 \mathrm{~s}$ at $200 \mathrm{kcps}$. All elements were quantified by direct measurement, except oxygen quantified by stoichiometry. As shown in Figure 1, there is good agreement between the measured and nominal concentrations at both accelerating voltages and ICRs. The relative standard deviations approach the rule of thumb value of $2 \%$, usually quoted as the accuracy of EPMA performed by WDS. The analytical totals are all within 95 and 105\%. While more validation is always needed by more users and with more reference materials, the described protocol and results confirm that standardless quantification enables any microscopist to perform accurate and fast EPMA.

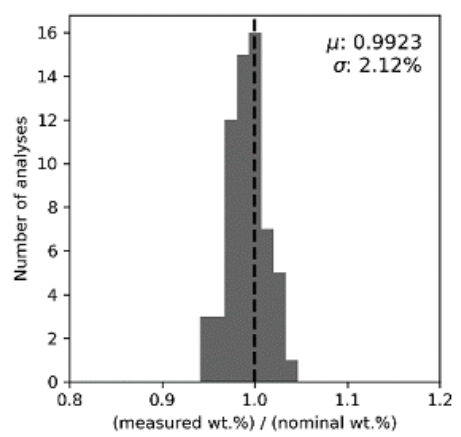

(a) $20 \mathrm{kV}, 50 \mathrm{kcps}$

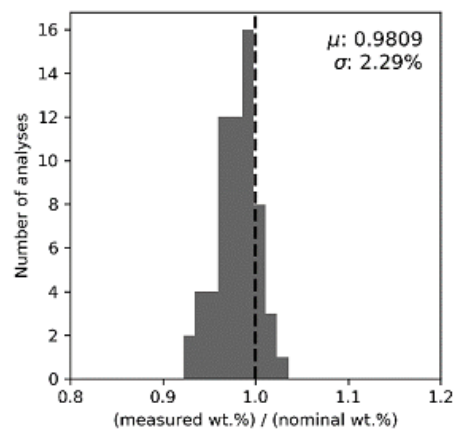

(b) $20 \mathrm{kV}, 200 \mathrm{kcps}$

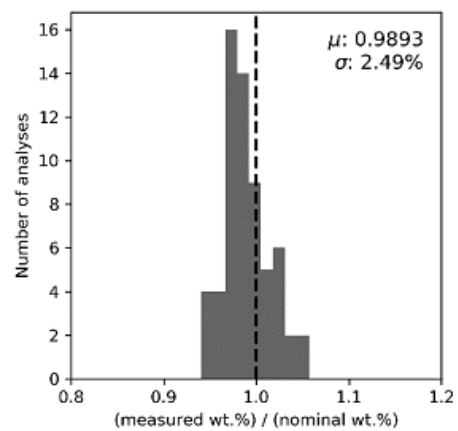

(c) $15 \mathrm{kV}, 50 \mathrm{kcps}$ 
Figure 1. Quantification results for three combinations of accelerating voltages and input count rates: (a) $20 \mathrm{kV}, 50 \mathrm{kcps}$, (b) $20 \mathrm{kV}, 200 \mathrm{kcps}$, and (c) $15 \mathrm{kV}, 50 \mathrm{kcps}$. The results are evaluated by taking the ratio between the measured and nominal concentration. A value close to unity indicates a good agreement. The average and relative standard deviation are shown next to each distribution.

References

[1] JI Goldstein et al, "Scanning Electron Microscopy and X-Ray Microanalysis", Springer (2018)

[2] PJ Statham, "Quantitative X-ray Energy Spectrometry”, University of Cambridge (1975)

[3] PJ Statham, Microsc. Microanal. 15 S2 (2009), 528

[4] PJ Statham, Microsc. Microanal. 20 S3 (2014), 690

[5] S Burgess et al, Microsc. Microanal. 13 S2 (2007), 1432

[6] P Pinard et al, EMAS 2019 Proceedings (2019), 269

[7] JL Pouchou and F Pichoir, "Quantitative analysis of homogeneous or stratified microvolumes applying the model PAP”, Electron Probe Quantitation, Plenum Press (1991), 31

[8] PJ Statham et al, IOP Conf. Ser.: Mater. Sci. Eng 109 (2016), 012016 